\title{
Outflow Velocities in Polar Coronal Holes
}

\author{
A. H. Gabriel ${ }^{1}$, F. Bely-Dubau ${ }^{2}$, P. Lemaire ${ }^{1}$ and E. Antonucci ${ }^{3}$ \\ ${ }^{1}$ Institut d'Astrophysique Spatiale, 91405 Orsay Cedex, France \\ ${ }^{2}$ Observatoire de la Côte d'Azur, BP4229, 06304 Nice Cedex, France \\ ${ }^{3}$ Observatorio Astronomico di Torino, I-10025 Pino Torinese, Italy
}

\begin{abstract}
Observations have been carried out using the oxygen VI multiplet ratio 1032/1038 $\AA$ from SUMER on SOHO. Analysis based on the Doppler dimming method shows that the outflow velocity in polar plumes is higher than that in the interplume region, contrary to many published suggestions. The addition of UVCS data for the interplume region, leads to a conclusion that the effective oxygen ion "temperature" in the radial direction has to rise to around $14 \mathrm{MK}$ over the height range 1.5 to 1.8 $\mathrm{R}_{\odot} \cdot$
\end{abstract}

\section{Introduction}

Plumes within polar coronal holes are observed at their best during the minimum phase of activity, when the Sun exhibits a simple dipole magnetic structure. For this reason, they feature in observations made during the 12 months following the launch of SOHO in December 1995. We are here specifically concerned with the plumes observed in spectral lines in the ultraviolet, characteristic of coronal temperatures around $1 \mathrm{MK}$. They are clearly detected in the $171 \AA$ Fe IX channel of the EIT imager, as well as in the 1032/1038 $\AA$ multiplet of O VI, observed by both SUMER and UVCS instruments. An early report (DeForest et al. 1977) claimed that plumes can be traced outwards from these UV measurements and are contiguous with white light plumes seen out to much greater distances. More recent work (Gabriel et al. 2003) has cast some doubt on this claim. In any case it is the UV plumes detected out to around $2 R_{\odot}$ that we are concerned with in the present contribution.

Many of the early papers on SOHO plumes discussed their possible physical properties and "suggested" that the wind outflow velocity is smaller in the plume areas than in the interplume areas. Only one observation (Giordano et al. 2000) claimed to have made an actual measurement of these flows, using the Doppler dimming technique from UVCS measurements on the O VI multiplet. This supported the above suggestion, although the error bars were rather large.

The resolution of this question of the role of plumes in the solar fast wind onset is quite important for the physics of these regions. Believing that there could be some doubt over what was becoming an accepted interpretation, Gabriel et al. (2003, hereafter referred to as GBL) set out to measure the outflow velocities, using the Doppler dimming technique on the O VI observations from 


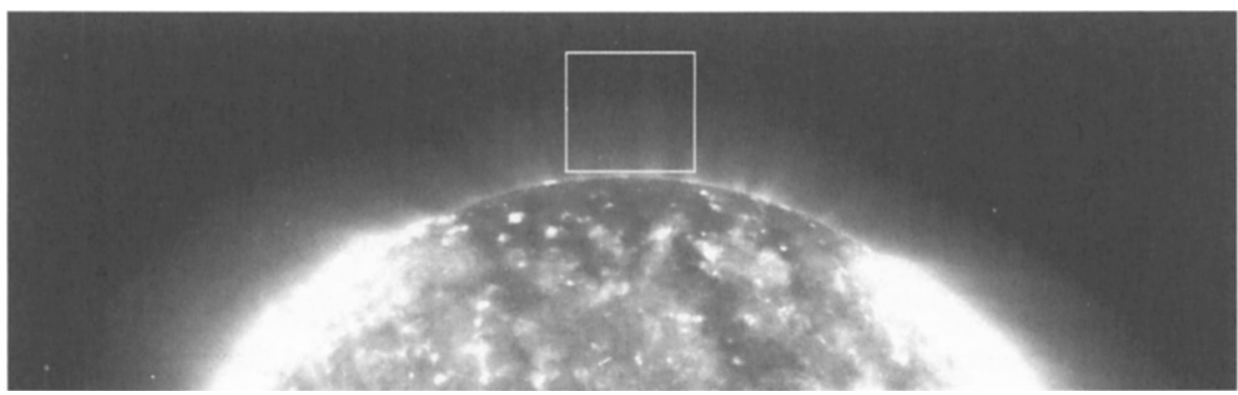

Figure 1. EIT image of the polar coronal hole, recorded at $171 \AA$. The superposed white rectangle shows the position of the coronal raster recorded by SUMER.

SUMER at lower heights. They exploited a unique data set which had been recorder on 1996 May 21 with the aim of measuring the electron temperature gradient in the polar coronal hole. Their results, summarized below, showed that, contrary to some accepted ideas, the wind velocities were indeed greater in the plume than in the interplume region. At about the same time, Teriaca et al. (2003, hereafter referred to as TPRB) published the analysis of another data set of June 1996, from SUMER and UVCS, and claimed that their interpretation favoured the more accepted view that the wind velocity was slower in the plume regions.

We are not yet ready to offer an explanation of why these two studies lead to conflicting interpretations, although this is an important remaining question. Rather, in this present contribution, we propose to explain some of the difficulties inherent in these types of interpretation and to outline some of the rigorous precautions that have been followed in the GBL investigations. We also give some preliminary results concerning the UVCS observations carried out simultaneously with the GBL studies.

\section{Plume results from SUMER observations}

We summarize briefly the results of the investigation by GBL. Figure 1 shows the region covered by the SUMER raster during the JOP2 campaign. In addition, data was recorded nearby on the solar disk for use in the stray light correction. Following instrumental calibration and stray light correction, the resultant intensity for O VI $1032 \AA$ is shown in Figure 2 as a function of height in the corona. The strong plume and weak interplume regions are marked as $B$ and A. Shown on the right of Figure 2 are the O VI multiplet intensity ratio used in the Doppler dimming method. The points shown are the result of some pixel rebinning, but are independent statistically, so that their scatter is a true indication of the very small random error in this data. This remarkable quality and separation of the plume and interplume results give confidence to the significance of the observations. For details, the reader is referred to the GBL paper, but here we show the results for the outflow velocities in Figure 3. The interplume region shows a normal acceleration with the velocity increasing with 

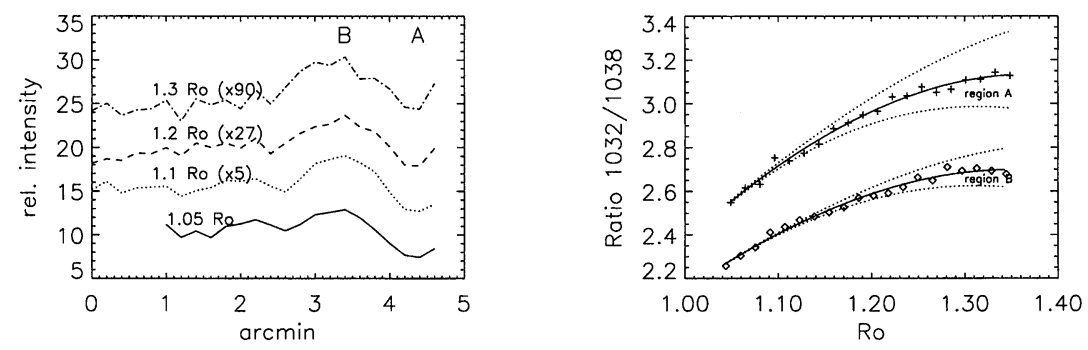

Figure 2. (left) The intensity of the coronal oxygen VI $1032 \AA$ line is here plotted against $\mathrm{E}-\mathrm{W}$ position for four radial distances, in arbitrary units. The intensities have been scaled by the factors shown, for convenience of plotting. (right) Observed coronal oxygen VI multiplet intensity ratio, after calibration and stray light correction, is plotted here as a function of radial distance, for the two regions indicated in the left-hand figure. The dotted curves show the limits obtained if there were an error of a factor $10 \%$ in the stray-light correction.

height. Results for the plume region depend on a parameter $F$, which is the unknown fraction of the line-of-sight occupied by the plume material. Independent of the precise value of $F$, the plume velocities are greater than the interplume and are approximately constant throughout this height range. There is a suggestion that the two velocities may converge in the range 1.5 to $2.0 \mathrm{R}_{\odot}$, in which case we might expect the plume structure to disappear at greater heights.

\section{Some critical points in the interpretation}

Here we draw attention to a number of difficulties in this approach, which have received special attention:-

Data-sets The only available sets in the SUMER archive, of long-exposure rasters at the limb using the O VI multiplet during solar minimum, are the JOP2 sequence used here and the June 1996 sequences used by TPRB. The JOP2 sequence is superior on account of its longer total exposure time (14 hours) and the fact that the spacecraft had been rolled through $90^{\circ}$ in order to optimize the stray light contribution. After November 1996, a malfunction in the SUMER raster excludes the possibility of further sequences of this type.

Stray light correction As for other workers, this has involved comparison between disk and above-limb exposures. However, in the present case, the point spread function has been used, and we have not assumed that the stray light will be the same for optically thick disk lines as for optically thin limb-brightened lines (see GBL). Neglecting this point can lead to important errors.

Interplume region It is important to start with a real interplume region, where the line-of-sight can be assumed to traverse a spherically symmetric atmosphere. 


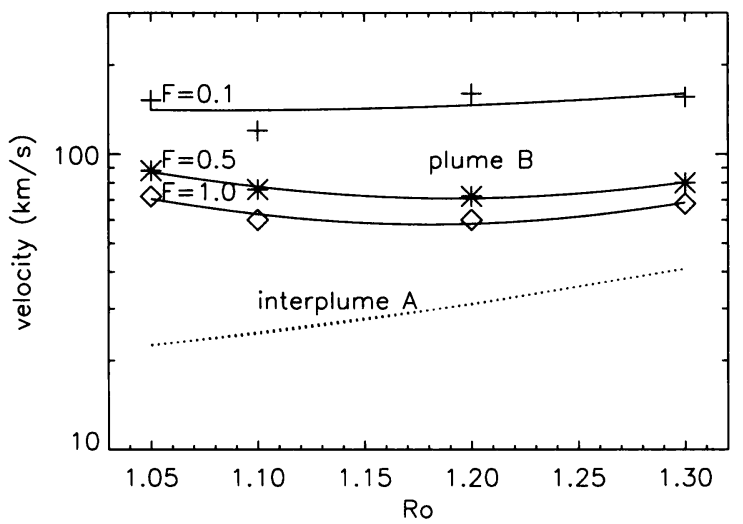

Figure 3. The derived outflow velocity for the plume B as a function of the radial distance and the line-of-sight parameter $F$. The interplume outflow is shown here as a dotted curve.

If this turns out to be a weaker plume region, then errors will be introduced into the analysis.

The 3rd dimension of the plume This is an unknown quantity which must be parameterized (our quantity $F$ ). If $F<1$, then the observed multiplet ratios will be a combination of $F$ of plume and $(1-F)$ of interplume material.

Electron densities A strategy for deducing a good interplume density value is essential in the interpretation. Here we assume that values for the line-ofsight mean density may be different from local values, based on spectroscopic line ratios. Figure 4 shows our two assumed density models. Also shown is a range of nine model variations, with conservation of outward mass flow, showing how the observed data points can serve to fix both density and outflow rates. Super-radial expansion has been allowed for, using Munro and Jackson (1977).

The Doppler dimming theory We have added many refinements to this theory. The most significant is to take account of the limb brightening (pumping) of the illuminating source from the solar transition region. This effect makes an important difference to the measured velocities and is, we believe, absent from all of the published models.

\section{Extension to include UVCS interplume data}

The JOP2 sequence in May 1996 included a range of UVCS measurements. Some of these have been analyzed and published (Antonucci et al. 2000), but not yet for the plume characteristics. We have here included a preliminary analysis of the UVCS data for our interplume region A. Figure 5 shows how these fit, together with our SUMER data, to a theoretical Doppler dimming model having a constant mass outflow equivalent to $27 \mathrm{~km} / \mathrm{s}$ at $1.1 \mathrm{R}_{\odot}$. Although the lower SUMER region fits well using an effective oxygen ion "temperature" equal to 

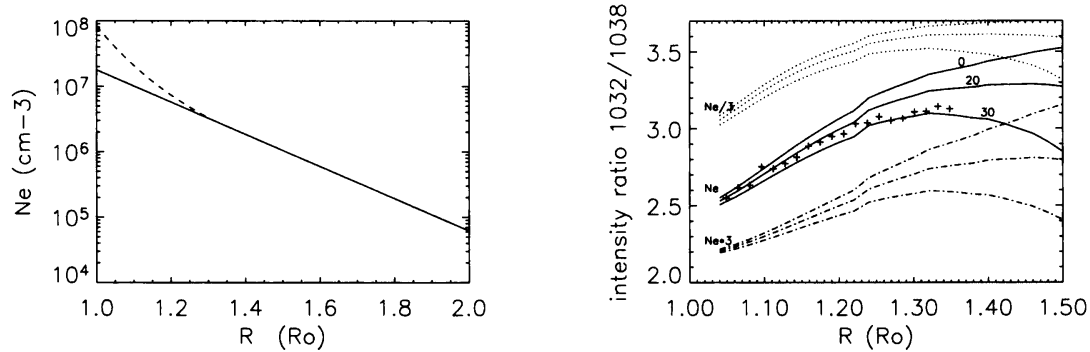

Figure 4. On the left are the adopted models for the electron density above a polar coronal hole. The full curve is a Thomson scattering mean density from Koutchmy (1994). The dotted curve is a local value from spectral line ratio diagnostics (Doschek et al. 1997). On the right are a family of 9 multiplet ratio models based on conservation of mass outflow, showing the observed SUMER data points (see text).

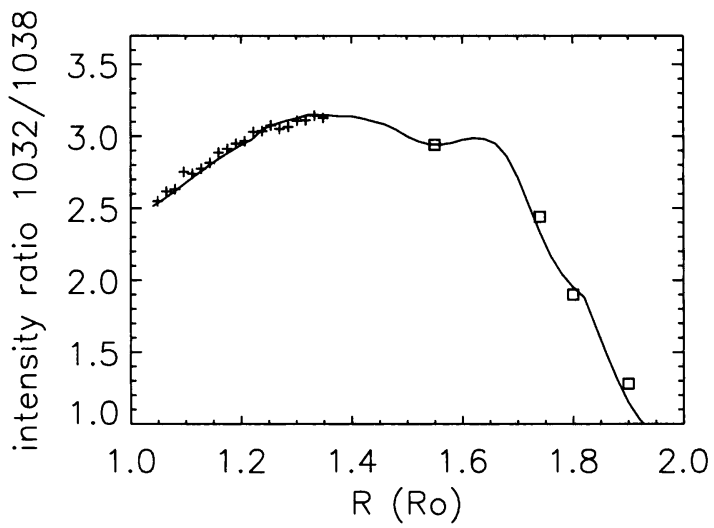

Figure 5. Showing the fit between theory and observations for the multiplet intensity ratio in the interplume region A. Crosses are the SUMER data points, while the small squares indicate UVCS observations 
the electron temperature of $1 \mathrm{MK}$, it has been found necessary to adopt an increasing ion temperature above $1.5 \mathrm{R}_{\odot}$ in order to fit the outer UVCS points, rising to $14 \mathrm{MK}$ at $1.8 \mathrm{R}_{\odot}$. This is in general agreement with the earlier analysis of Antonucci et al. (2000).

\section{References}

Antonucci, E., Dodero, M. A., \& Giordano, S. 2000, Solar Phys., 197, 115

DeForest, C. E., Hoeksema, J. E., Gurman, J. B., Thompson, B. J., Plunkett, S. P., Howard, R., Harrison, R. C., \& Hassler, D. M. 1997, Solar Phys., 175,393

Doschek, G. A., et al. 1997, ApJ, 482, L109

Gabriel, A. H., Bely-Dubau, F., \& Lemaire, P. 2003, ApJ, 589, 623, (hereafter GBL)

Giordano, S., Antonucci, E., Noci, G., Romoli, M, Kohl, J. L., 2000, ApJ, 531, L79

Koutchmy, S. 1994, Adv. in Space Res., 14, 29

Munro, R. H., \& Jackson, B. V. 1977, ApJ, 213, 847

Teriaca, L., Poletto, G., Romoli, M., \& Biesecker, D. A. 2003, ApJ, 588, 566, (hereafter TPRB) 